\title{
Shifting and Variational Properties for Fourier-Feynman Transform and Convolution
}

\author{
Byoung Soo Kim \\ School of Liberal Arts, Seoul National University of Science and Technology, Seoul 01811, Republic of Korea \\ Correspondence should be addressed to Byoung Soo Kim; mathkbs@seoultech.ac.kr
}

Received 5 June 2015; Accepted 10 August 2015

Academic Editor: Dashan Fan

Copyright (C) 2015 Byoung Soo Kim. This is an open access article distributed under the Creative Commons Attribution License, which permits unrestricted use, distribution, and reproduction in any medium, provided the original work is properly cited.

Shifting, scaling, modulation, and variational properties for Fourier-Feynman transform of functionals in a Banach algebra $\mathcal{S}$ are given. Cameron and Storvick's translation theorem can be obtained as a corollary of our result. We also study shifting, scaling, and modulation properties for the convolution product of functionals in $\mathcal{S}$.

\section{Introduction}

Let $C_{0}[0, T]$ denote the Wiener space, that is, the space of real valued continuous functions $x$ on $[0, T]$ with $x(0)=0$. The concept of $L_{1}$ analytic Fourier-Feynman transform for functionals on Wiener space was introduced by Brue in [1]. In [2], Cameron and Storvick introduced $L_{2}$ analytic FourierFeynman transform. In [3], Johnson and Skoug developed $L_{p}$ analytic Fourier-Feynman transform for $1 \leq p \leq 2$ that extended the results in [2].

In $[4,5]$, Huffman et al. defined a convolution product for functionals on Wiener space and showed that the Fourier-Feynman transform of a convolution product is a product of Fourier-Feynman transforms. Recently Kim et al. [6] obtained change of scale formulas for Wiener integrals related to Fourier-Feynman transform and convolution. For a detailed survey of the previous work on Fourier-Feynman transform and related topics, see [7].

Let $\mathscr{M}$ denote the class of all Wiener measurable subsets of $C_{0}[0, T]$ and let $m$ denote Wiener measure. Then $\left(C_{0}[0, T], \mathscr{M}, m\right)$ is a complete measure space and we denote the Wiener integral of a functional $F$ by

$$
\int_{C_{0}[0, T]} F(x) d m(x) .
$$

A subset $E$ of $C_{0}[0, T]$ is said to be scale-invariant measurable [8] provided $\rho E$ is measurable for each $\rho>0$, and a scale-invariant measurable set $N$ is said to be scale-invariant null provided $m(\rho N)=0$ for each $\rho>0$. A property that holds except on a scale-invariant null set is said to hold scaleinvariant almost everywhere (s-a.e.).

Let $\mathbb{C}_{+}$and $\mathbb{C}_{+}^{\sim}$ denote the sets of complex numbers with positive real part and the complex numbers with nonnegative real part, respectively. Let $F$ be a complex valued measurable functional on $C_{0}[0, T]$ such that the Wiener integral

$$
J_{F}(\lambda)=\int_{C_{0}[0, T]} F\left(\lambda^{-1 / 2} x\right) d m(x)
$$

exists as a finite number for all $\lambda>0$. If there exists a function $J_{F}^{*}(\lambda)$ analytic in $\mathbb{C}_{+}$such that $J_{F}^{*}(\lambda)=J_{F}(\lambda)$ for all $\lambda>0$, then $J_{F}^{*}(\lambda)$ is defined to be the analytic Wiener integral of $F$ over $C_{0}[0, T]$ with parameter $\lambda$, and for $\lambda \in \mathbb{C}_{+}$we write

$$
\int_{C_{0}[0, T]}^{\mathrm{anw} \mathrm{w}_{\lambda}} F(x) d m(x)=J_{F}^{*}(\lambda) .
$$

If the following limit exists for nonzero real $q$, then we call it the analytic Feynman integral of $F$ over $C_{0}[0, T]$ with parameter $q$ and we write

$$
\int_{C_{0}[0, T]}^{\operatorname{anf}_{q}} F(x) d m(x)=\lim _{\lambda \rightarrow-i q} \int_{C_{0}[0, T]}^{\mathrm{anw}_{\lambda}} F(x) d m(x),
$$

where $\lambda$ approaches $-i q$ through $\mathbb{C}_{+}$.

Now we will introduce the class of functionals that we work with in this paper. The Banach algebra $\mathcal{S}$, which 
was introduced by Cameron and Storvick [9], consists of functionals expressible in the form

$$
F(x)=\int_{L_{2}[0, T]} \exp \{i\langle v, x\rangle\} d f(v)
$$

for s-a.e. $x$ in $C_{0}[0, T]$, where the associated measure $f$ is a complex Borel measure on $L_{2}[0, T]$ and $\langle v, x\rangle$ denote the Paley-Wiener-Zygmund stochastic integral $\int_{0}^{T} v(t) d x(t)$.

In this paper, we study shifting, scaling, modulation, and variational properties for Fourier-Feynman transform of functionals in $\mathcal{S}$. Shifting properties are some of the important properties of Fourier transform. In Section 2, we develop shifting properties for Fourier-Feynman transform. For example, time shifting, frequency shifting, scaling, and modulation properties for Fourier-Feynman transform are given.

In Section 3, we study variational properties for FourierFeynman transform of functionals in $\mathcal{S}$ and in the last section we develop shifting, scaling, and modulation properties for convolution product of functionals in $\mathcal{S}$.

The Banach algebra $\mathcal{S}$ is a very rich class of functionals. It is known that important functionals in quantum mechanics and Feynman integration theory belong to $\mathcal{S}$. For example, functionals of the form

$$
F(x)=\exp \left\{\int_{0}^{T} \int_{0}^{T} f(s, t, x(s), x(t)) d s d t\right\}
$$

were discussed in the book by Feynman and Hibbs [10] on path integrals and in Feynman's original paper [11]. For appropriate $f:[0, T]^{2} \times \mathbb{R}^{2} \rightarrow \mathbb{C}$, functionals of form (6) are known to belong to $\mathcal{S}$ [12]. Hence the results in this paper can be immediately applied to many functionals of form (6).

\section{Shifting Properties for Fourier-Feynman Transform}

In this section we develop some of the important properties relevant to shifting (translating) and computational rules for Fourier-Feynman transform of functionals in the Banach algebra $\delta$. Let us begin with the definition of the FourierFeynman transform of functionals on Wiener space.

Let $1 \leq p<\infty$ and let $q$ be a nonzero real number throughout this paper.

Definition 1. Let $F$ be a functional on $C_{0}[0, T]$. For $\lambda \in \mathbb{C}_{+}$ and $y \in C_{0}[0, T]$, let

$$
T_{\lambda}[F](y)=\int_{C_{0}[0, T]}^{\mathrm{anw}_{\lambda}} F(x+y) d m(x)
$$

For $1<p<\infty$, we define $L_{p}$ analytic Fourier-Feynman transform $T_{q}^{(p)}[F]$ of $F$ on $C_{0}[0, T]$ by the formula $\left(\lambda \in \mathbb{C}_{+}\right)$

$$
T_{q}^{(p)}[F](y)=\underset{\lambda \rightarrow-i q}{\lim . T_{\lambda}}[F](y),
$$

whenever this limit exists; that is, for each $\rho>0$,

$$
\begin{aligned}
& \lim _{\lambda \rightarrow-i q} \int_{C_{0}[0, T]}\left|T_{\lambda}[F](\rho x)-T_{q}^{(p)}[F](\rho x)\right|^{p^{\prime}} d m(x) \\
& \quad=0
\end{aligned}
$$

where $1 / p+1 / p^{\prime}=1$. We define $L_{1}$ analytic Fourier-Feynman transform $T_{q}^{(1)}[F]$ of $F$ by $\left(\lambda \in \mathbb{C}_{+}\right)$

$$
T_{q}^{(1)}[F](y)=\lim _{\lambda \rightarrow-i q} T_{\lambda}[F](y),
$$

for s-a.e. $y \in C_{0}[0, T]$, whenever this limit exists [2-5].

Since $T_{\lambda}$ is linear, obviously $T_{q}^{(p)}$ is linear; that is,

$$
T_{q}^{(p)}[a F+b G](y)=a T_{q}^{(p)}[F](y)+b T_{q}^{(p)}[G](y)
$$

for all constants $a, b$ and functionals $F, G$ on $C_{0}[0, T]$, whenever each transform exists.

By Definition (4) of the analytic Feynman integral and $L_{1}$ analytic Fourier-Feynman transform (10), it is easy to see that

$$
T_{q}^{(1)}(F)(y)=\int_{C_{0}[0, T]}^{\mathrm{anf}_{q}} F(x+y) d m(x) .
$$

In particular, if $F \in \mathcal{S}$, then $F$ is analytic Feynman integrable and

$$
T_{q}^{(1)}(F)(0)=\int_{C_{0}[0, T]}^{\operatorname{anf}_{q}} F(x) d m(x) .
$$

Huffman et al. established the existence of FourierFeynman transform on $C_{0}[0, T]$ for functionals in $\mathcal{S}$.

Theorem 2 (Theorem 3.1 of [5]). Let $F \in \mathcal{S}$ be given by (5). Then for all $\lambda \in \mathbb{C}_{+}$

$$
T_{\lambda}[F](y)=\int_{L_{2}[0, T]} \exp \left\{i\langle v, y\rangle-\frac{1}{2 \lambda}\|v\|^{2}\right\} d f(v)
$$

for s-a.e. $y \in C_{0}[0, T]$. Moreover the Fourier-Feynman transform $T_{q}^{(p)}[F]$ exists, belongs to $\mathcal{S}$, and is given by

$$
T_{q}^{(p)}[F](y)=\int_{L_{2}[0, T]} \exp \left\{i\langle v, y\rangle-\frac{i}{2 q}\|v\|^{2}\right\} d f(v)
$$

for s-a.e. $y \in C_{0}[0, T]$.

The Fourier transform $\mathscr{F}$ turns a function $f$ into a new function $\mathscr{F}[f]$. Because the transform is used in signal analysis, we usually use $t$ for time as the variable with $f$ and $\omega$ as the variable of the transform $\mathscr{F}[f]$; that is,

$$
\mathscr{F}[f](\omega)=\int_{-\infty}^{\infty} f(t) e^{-i \omega t} d t
$$

Engineers refer to the variable $\omega$ in the transformed function as the frequency of the signal $f[13]$. 
We will use the same convention in this paper; that is, for a Fourier-Feynman transform $T_{q}^{(p)}[F](y)$ of $F(x)$, we call the variable $x$ a time and the variable $y$ a frequency.

Our first result in this section shows that the time shifting of the Fourier-Feynman transform is equal to the frequency shifting of the Fourier-Feynman transform.

Theorem 3. Let $F$ be a functional on $C_{0}[0, T]$ and let $x_{0} \in$ $C_{0}[0, T]$. Then one has

$$
T_{q}^{(p)}\left[F\left(\cdot-x_{0}\right)\right](y)=T_{q}^{(p)}[F]\left(y-x_{0}\right)
$$

if all sides exist.

Proof. For all $\lambda>0$ and for s-a.e. $y \in C_{0}[0, T]$,

$$
\begin{aligned}
T_{\lambda} & {\left[F\left(\cdot-x_{0}\right)\right](y) } \\
& =\int_{C_{0}[0, T]} F\left(\lambda^{-1 / 2} x-x_{0}+y\right) d m(x) \\
& =T_{\lambda}[F]\left(y-x_{0}\right)
\end{aligned}
$$

if the Wiener integral exists. Hence we have the result.

The following theorem is reminiscent of the time shifting theorem for the Fourier transform. Hence we call the following theorem the time shifting formula for Fourier-Feynman transform on Wiener space. It says that if we shift back $x_{0}$ and replace $F(x)$ by $F\left(x-x_{0}\right)$, then the Fourier-Feynman transform of this shifted function is equal to the FourierFeynman transform of $F(x) \exp \left\{i q\left\langle x_{0}, x\right\rangle\right\}$ multiplied by an exponential factor.

Theorem 4 (time shifting). Let $F \in \mathcal{S}$ be given by (5) and let $x_{0} \in C_{0}[0, T]$. Then one has

$$
\begin{gathered}
T_{q}^{(p)}\left[F\left(\cdot-x_{0}\right)\right](y)=\exp \left\{-i q\left\langle x_{0}, y\right\rangle+\frac{i q}{2}\left\|x_{0}\right\|^{2}\right\} \\
\cdot T_{q}^{(p)}\left[F(\cdot) \exp \left\{i q\left\langle x_{0}, \cdot\right\rangle\right\}\right](y)
\end{gathered}
$$

for s-a.e. $y \in C_{0}[0, T]$.

Proof. Let $G(x)=F(x) \exp \left\{i q\left\langle x_{0}, x\right\rangle\right\}$. Using (5) we write $G(x)$ as

$$
\begin{aligned}
G(x) & =\int_{L_{2}[0, T]} \exp \left\{i\left\langle v+q x_{0}, x\right\rangle\right\} d f(v) \\
& =\int_{L_{2}[0, T]} \exp \{i\langle w, x\rangle\} d g(w),
\end{aligned}
$$

where $g(E)=f\left(E-q x_{0}\right)$ for a Borel subset $E$ of $L_{2}[0, T]$; that is, $G$ also belongs to $\mathcal{S}$. Now by Theorem 2, we have

$$
\begin{gathered}
T_{q}^{(p)}[G](y)=\int_{L_{2}[0, T]} \exp \{i\langle w, y\rangle \\
\left.-\frac{i}{2 q}\|w\|^{2}\right\} d g(w)
\end{gathered}
$$

$$
\begin{aligned}
& =\int_{L_{2}[0, T]} \exp \left\{i\left\langle v+q x_{0}, y\right\rangle\right. \\
& \left.-\frac{i}{2 q}\left\|v+q x_{0}\right\|^{2}\right\} d f(v)=\exp \left\{i q\left\langle x_{0}, y\right\rangle\right. \\
& \left.-\frac{i q}{2}\left\|x_{0}\right\|^{2}\right\} \int_{L_{2}[0, T]} \exp \left\{i\left\langle v, y-x_{0}\right\rangle\right. \\
& \left.-\frac{i}{2 q}\|v\|^{2}\right\} d f(v) .
\end{aligned}
$$

Finally by Theorem 2 again we have

$$
\begin{aligned}
& T_{q}^{(p)}[G](y) \\
& \quad=\exp \left\{i q\left\langle x_{0}, y\right\rangle-\frac{i q}{2}\left\|x_{0}\right\|^{2}\right\} T_{q}^{(p)}[F]\left(y-x_{0}\right)
\end{aligned}
$$

and so by (17) the proof is complete.

Cameron and Storvick [14] presented a new translation theorem for the analytic Feynman integral on Wiener space. Moreover Ahn et al. [15] gave a simple proof of an abstract Wiener space version of the translation theorem. Taking $p=$ 1 and $y=0$ in (19) and considering (13) we obtain Cameron and Storvick's translation theorem as follows. Hence Theorem 4 can be viewed as Cameron and Storvick's translation theorem for the Fourier-Feynman transform.

Corollary 5. Let $F \in \mathcal{S}$ be given by (5) and let $x_{0} \in C_{0}[0, T]$. Then one has

$$
\begin{gathered}
\int_{C_{0}[0, T]}^{a n f_{q}} F\left(x-x_{0}\right) d m(x)=\exp \left\{\frac{i q}{2}\left\|x_{0}\right\|^{2}\right\} \\
\cdot \int_{C_{0}[0, T]}^{a n f_{q}} F(x) \exp \left\{i q\left\langle x_{0}, x\right\rangle\right\} d m(x) .
\end{gathered}
$$

Next theorem is reminiscent of the frequency shifting theorem for the Fourier transform. Using Theorem 3 we have the following property for the frequency shifting of the Fourier-Feynman transform.

Theorem 6 (frequency shifting). Let $F \in \mathcal{S}$ be given by (5) and let $y_{0} \in C_{0}[0, T]$. Then one has

$$
\begin{aligned}
T_{q}^{(p)}[F]\left(y-y_{0}\right)= & \exp \left\{-i q\left\langle y_{0}, y\right\rangle+\frac{i q}{2}\left\|y_{0}\right\|^{2}\right\} \\
& \cdot T_{q}^{(p)}\left[F(\cdot) \exp \left\{i q\left\langle y_{0}, \cdot\right\rangle\right\}\right](y)
\end{aligned}
$$

for s-a.e. $y \in C_{0}[0, T]$.

The following theorem is called a scaling theorem because we want the transform not of $F(x)$, but of $F(a x)$, in which $a$ can be thought of as a scaling factor.

Theorem 7 (scaling). Let $F \in \mathcal{S}$ be given by (5) and let a be a nonzero real number. Then one has

$$
T_{q}^{(p)}[F(a \cdot)](y)=T_{q / a^{2}}^{(p)}[F](a y)
$$

for s-a.e. $y \in C_{0}[0, T]$. 
Proof. Let $G(x)=F(a x)$ for $x \in C_{0}[0, T]$. Using (5) we can write $G(x)$ as

$$
\begin{aligned}
G(x) & =\int_{L_{2}[0, T]} \exp \{i\langle v, a x\rangle\} d f(v) \\
& =\int_{L_{2}[0, T]} \exp \{i\langle w, x\rangle\} d g(w),
\end{aligned}
$$

where $g(E)=f(E / a)$ for each Borel subset $E$ of $L_{2}[0, T]$. By Theorem 2, we have

$$
\begin{aligned}
T_{q}^{(p)}[G](y) & \\
= & \int_{L_{2}[0, T]} \exp \left\{i\langle w, y\rangle-\frac{i}{2 q}\|w\|^{2}\right\} d g(w) \\
& =\int_{L_{2}[0, T]} \exp \left\{i\langle v, a y\rangle-\frac{a^{2} i}{2 q}\|v\|^{2}\right\} d f(v)
\end{aligned}
$$

for s-a.e. $y \in C_{0}[0, T]$. Finally by Theorem 2 again we obtain the result.

Our next corollary follows immediately from the scaling theorem above by putting $a=-1$. This result is called time reversal because we replace $x$ by $-x$ in $F(x)$ to get $F(-x)$. The transform of this new functional is obtained by simply replacing $y$ by $-y$ in the transform of $F(x)$.

Corollary 8 (time reversal). Let $F \in \mathcal{S}$ be given by (5). Then one has

$$
T_{q}^{(p)}[F(-\cdot)](y)=T_{q}^{(p)}[F](-y)
$$

for s-a.e. $y \in C_{0}[0, T]$.

Our next theorem is useful in obtaining the FourierFeynman transforms of new functionals from the FourierFeynman transforms of old functionals for which we know their Fourier-Feynman transform.

Theorem 9 (modulation). Let $F \in \mathcal{S}$ be given by (5) and let $x_{0} \in C_{0}[0, T]$. Then one has

$$
\begin{aligned}
T_{q}^{(p)} & {\left[F(\cdot) \cos \left(q\left\langle x_{0}, \cdot\right\rangle\right)\right](y) } \\
= & \frac{1}{2}\left(K[F]\left(x_{0}, y\right)+K[F]\left(-x_{0}, y\right)\right), \\
T_{q}^{(p)} & {\left[F(\cdot) \sin \left(q\left\langle x_{0}, \cdot\right\rangle\right)\right](y) } \\
& =\frac{1}{2 i}\left(K[F]\left(x_{0}, y\right)-K[F]\left(-x_{0}, y\right)\right),
\end{aligned}
$$

where

$$
\begin{aligned}
K & {[F]\left(x_{0}, y\right) } \\
& =\exp \left\{i q\left\langle x_{0}, y\right\rangle-\frac{i q}{2}\left\|x_{0}\right\|^{2}\right\} T_{q}^{(p)}\left[F\left(\cdot-x_{0}\right)\right](y)
\end{aligned}
$$

for s-a.e. $y \in C_{0}[0, T]$.
Proof. Put $\cos \left(q\left\langle x_{0}, \cdot\right\rangle\right)=(1 / 2)\left(\exp \left\{i q\left\langle x_{0}, \cdot\right\rangle\right\}+\exp \left\{-i q\left\langle x_{0}\right.\right.\right.$, $\cdot\rangle\})$ and use the linearity of the Fourier-Feynman transform $T_{q}^{(p)}$ to get

$$
\begin{aligned}
T_{q}^{(p)} & {\left[F(\cdot) \cos \left(q\left\langle x_{0}, \cdot\right\rangle\right)\right](y) } \\
& =\frac{1}{2}\left(T_{q}^{(p)}\left[F(\cdot) \exp \left\{i q\left\langle x_{0}, \cdot\right\rangle\right\}\right](y)\right. \\
& \left.+T_{q}^{(p)}\left[F(\cdot) \exp \left\{-i q\left\langle x_{0}, \cdot\right\rangle\right\}\right](y)\right) .
\end{aligned}
$$

Finally by the time shifting theorem or frequency shifting theorem we obtain (29). Using $\sin \left(q\left\langle x_{0}, \cdot\right\rangle\right)=(1 / 2 i)\left(\exp \left\{i q\left\langle x_{0}\right.\right.\right.$, $\left.\cdot\rangle\}-\exp \left\{-i q\left\langle x_{0}, \cdot\right\rangle\right\}\right)$ the second conclusion is proved similarly.

Since the Dirac measure concentrated at $v=0$ in $L_{2}[0, T]$ is a complex Borel measure, the constant function $F \equiv 1$ belongs to $\mathcal{S}$. Hence we have the following corollary.

Corollary 10. Let $x_{0} \in C_{0}[0, T]$. Then one has

$$
\begin{aligned}
T_{q}^{(p)} & {\left[\cos \left(q\left\langle x_{0}, \cdot\right\rangle\right)\right](y) } \\
= & \cos \left(q\left\langle x_{0}, y\right\rangle\right) \exp \left\{-\frac{i q}{2}\left\|x_{0}\right\|^{2}\right\}, \\
T_{q}^{(p)} & {\left[\sin \left(q\left\langle x_{0}, \cdot\right\rangle\right)\right](y) } \\
& =\sin \left(q\left\langle x_{0}, y\right\rangle\right) \exp \left\{-\frac{i q}{2}\left\|x_{0}\right\|^{2}\right\}
\end{aligned}
$$

for s-a.e. $y \in C_{0}[0, T]$.

Proof. Since $T_{q}^{(p)}\left[F\left(\cdot-x_{0}\right)\right](y)=T_{q}^{(p)}[F]\left(y-x_{0}\right)=1$ for $F \equiv 1$, by the modulation property, Theorem 9 , and Euler's formula, (33) and (34) follow immediately.

\section{Variational Properties for Fourier-Feynman Transform}

In using the Fourier transform to solve differential equations, we need an expression relating the transform of $f^{\prime}$ to that of $f$. In this section we develop similar relationships for FourierFeynman transform on Wiener space; that is, we provide variational properties for Fourier-Feynman transform of functionals in the Banach algebra $\mathcal{S}$.

Definition 11. Let $F$ be a functional on $C_{0}[0, T]$ and let $w_{1}, \ldots, w_{n} \in C_{0}[0, T]$. Then

$$
\delta F\left(x, w_{1}\right)=\left.\frac{\partial}{\partial t} F\left(x+t w_{1}\right)\right|_{t=0}
$$

(if it exists) is called the first variation of $F(x)$. The higher order variations of $F(x)$ are defined inductively. For example, the 2nd order variation of $F(x)$ is the first variation of $\delta F\left(x, w_{1}\right)$ with respect to $x$ and is defined by

$$
\delta^{2} F\left(x, w_{2} ; w_{1}\right)=\left.\frac{\partial}{\partial t} \delta F\left(x+t w_{2}, w_{1}\right)\right|_{t=0}
$$


and $n$th order variation is defined by

$$
\begin{aligned}
\delta^{n} F & \left(x, w_{n} ; \ldots ; w_{1}\right) \\
\quad= & \left.\frac{\partial}{\partial t} \delta^{n-1} F\left(x+t w_{n}, w_{n-1} ; \ldots ; w_{1}\right)\right|_{t=0}
\end{aligned}
$$

for $n=3,4, \ldots$. If $w=w_{1}=\cdots=w_{n}$, then we denote $\delta^{n} F(x, w ; \ldots ; w)$ as $\delta^{n} F(x, w)$.

Theorem 12. Let $F \in \mathcal{S}$ be given by (5) with $\int_{L_{2}[0, T]}\|v\|^{n}$. $d|f|(v)<\infty$. Then for s-a.e. $x$ and $w_{1}, \ldots, w_{n}$ in $C_{0}[0, T]$, $\delta^{n} F\left(x, w_{n} ; \ldots ; w_{1}\right)$ exists, is an element of $\mathcal{S}$ as a function of $x$, and is given by the formula

$$
\delta^{n} F\left(x, w_{n} ; \ldots ; w_{1}\right)=\int_{L_{2}[0, T]} \exp \{i\langle v, x\rangle\} d f_{n}(v),
$$

where $f_{n}$ is a complex Borel measure on $L_{2}[0, T]$ defined by

$$
f_{n}(E)=i^{n} \int_{E}\left\langle v, w_{1}\right\rangle \cdots\left\langle v, w_{n}\right\rangle d f(v)
$$

for each Borel subset $E$ of $C_{0}[0, T]$.

Proof. We will prove that

$$
\begin{aligned}
& \delta^{2} F\left(x, w_{2} ; w_{1}\right) \\
& \quad=i^{2} \int_{L_{2}[0, T]}\left\langle v, w_{1}\right\rangle\left\langle v, w_{2}\right\rangle \exp \{i\langle v, x\rangle\} d f(v)
\end{aligned}
$$

for s-a.e. $x$ and $w_{1}, w_{2}$ in $C_{0}[0, T]$. Using Lemma 3.1 of [16], we have

$$
\begin{gathered}
\delta^{2} F\left(x, w_{2} ; w_{1}\right)=\left.\frac{\partial}{\partial t}\left(\delta F\left(x+t w_{2}, w_{1}\right)\right)\right|_{t=0} \\
=\frac{\partial}{\partial t}\left(\int_{L_{2}[0, T]} i\left\langle v, w_{1}\right\rangle\right. \\
\left.\cdot \exp \left\{i\langle v, x\rangle+i t\left\langle v, w_{2}\right\rangle\right\} d f(v)\right)\left.\right|_{t=0} .
\end{gathered}
$$

Now the result follows if we can pass the differentiation under the integral sign. But this is done because by the Fubini theorem

$$
\begin{aligned}
& \int_{C_{0}[0, T]^{2}} \int_{L_{2}[0, T]} \mid\left\langle v, w_{1}\right\rangle \\
& \cdot\left\langle v, w_{2}\right\rangle|d| f \mid(v) d(m \times m)\left(w_{1}, w_{2}\right) \\
& =\frac{2}{\pi} \int_{L_{2}[0, T]}\|v\|^{2} d|f|(v)
\end{aligned}
$$

which is finite and so $\int_{L_{2}[0, T]}\left|\left\langle v, w_{1}\right\rangle\left\langle v, w_{2}\right\rangle\right| d|f|(v)<\infty$ for s-a.e. $w_{1}, w_{2}$ in $C_{0}[0, T]$. Now by mathematical induction we obtain general result (38).

If $w=w_{1}=\cdots=w_{n}$ in Theorem 12, then we have the following corollary.
Corollary 13. Let $F \in \mathcal{S}$ be given by (5) with $\int_{L_{2}[0, T]}\|v\|^{n}$. $d|f|(v)<\infty$. Then for s-a.e. $x$ and $w$ in $C_{0}[0, T], \delta^{n} F(x, w)$ exists, is an element of $\mathcal{S}$ as a function of $x$, and is given by the formula

$$
\delta^{n} F(x, w)=\int_{L_{2}[0, T]} \exp \{i\langle v, x\rangle\} d f_{n}(v),
$$

where $f_{n}$ is the complex Borel measure on $L_{2}[0, T]$ defined by

$$
f_{n}(E)=i^{n} \int_{E}\langle v, w\rangle^{n} d f(v)
$$

for each Borel subset $E$ of $C_{0}[0, T]$.

In our next theorem, for functionals in $\mathcal{S}$ we establish a relationship between the Fourier-Feynman transform of the variation and the variation of the Fourier-Feynman transform. Also see Corollary 4.3 of [15] for a similar result.

Theorem 14. Let $F \in \mathcal{S}$ be given by (5) with $\int_{L_{2}[0, T]}\|v\|^{n}$. $d|f|(v)<\infty$. Then one has

$$
\begin{aligned}
T_{q}^{(p)} & {\left[\delta^{n} F\left(\cdot, w_{n} ; \ldots ; w_{1}\right)\right](y) } \\
& =\delta^{n} T_{q}^{(p)}[F]\left(y, w_{n} ; \ldots ; w_{1}\right)
\end{aligned}
$$

for s-a.e. $y$ and $w_{1}, \ldots, w_{n}$ in $C_{0}[0, T]$. Also, both of the expressions in (45) are given by the expression

$$
\begin{aligned}
& i^{n} \int_{L_{2}[0, T]}\left\langle v, w_{1}\right\rangle \cdots\left\langle v, w_{n}\right\rangle \\
& \quad \cdot \exp \left\{i\langle v, y\rangle-\frac{i}{2 q}\|v\|^{2}\right\} d f(v)
\end{aligned}
$$

for s-a.e. $y$ and $w_{1}, \ldots, w_{n}$ in $C_{0}[0, T]$.

Proof. By (38) and Theorem 2, we have

$$
\begin{aligned}
T_{q}^{(p)} & {\left[\delta^{n} F\left(\cdot, w_{n} ; \ldots ; w_{1}\right)\right](y) } \\
& =\int_{L_{2}[0, T]} \exp \left\{i\langle v, y\rangle-\frac{i}{2 q}\|v\|^{2}\right\} d f_{n}(v)
\end{aligned}
$$

for s-a.e. $y$ and $w_{1}, \ldots, w_{n}$ in $C_{0}[0, T]$. Now by (39) we know that the last expression can be rewritten as (46). On the other hand, by the same method as in the proof of Theorem 12, we see that the right hand side of (45) is also expressed as (46).

Letting $w=w_{1}=\cdots=w_{n}$ in Theorem 14 we have the following corollary.

Corollary 15. Let $F \in \mathcal{S}$ be given by (5) with $\int_{L_{2}[0, T]}\|v\|^{n}$. $d|f|(v)<\infty$. Then one has

$$
\begin{aligned}
T_{q}^{(p)} & {\left[\delta^{n} F(\cdot, w)\right](y)=\delta^{n} T_{q}^{(p)}[F](y, w) } \\
= & i^{n} \int_{L_{2}[0, T]}\langle v, w\rangle^{n} \exp \left\{i\langle v, y\rangle-\frac{i}{2 q}\|v\|^{2}\right\} d f(v)
\end{aligned}
$$

for s-a.e. $y$ and $w$ in $C_{0}[0, T]$. 
The following theorem involves an iterated FourierFeynman transform of $n$th order variation.

Theorem 16. Let $F \in \mathcal{S}$ be given by (5) with $\int_{L_{2}[0, T]}\|v\|^{n}$. $d|f|(v)<\infty$. Then one has

$$
\begin{aligned}
T_{q}^{(p)} & {\left[\cdots T_{q}^{(p)}\left[\delta^{n} F(x, \cdot ; \ldots ; \cdot)\right]\left(z_{1}\right) \cdots\right]\left(z_{n}\right) } \\
& =\delta^{n} F\left(x, z_{n} ; \ldots ; z_{1}\right)
\end{aligned}
$$

for s-a.e. $x$ and $z_{1}, \ldots, z_{n}$ in $C_{0}[0, T]$.

Proof. Let $G(x, w)=\int_{L_{2}[0, T]}\langle v, w\rangle \exp \{i\langle v, x\rangle\} d f(v)$. For $\lambda>$ 0 , we obtain

$$
\begin{aligned}
T_{\lambda} & {[G(x, \cdot)](z)=\int_{C_{0}[0, T]} G\left(x, \lambda^{-1 / 2} w+z\right) d m(w) } \\
& =\int_{C_{0}[0, T]} \int_{L_{2}[0, T]}\left(\lambda^{-1 / 2}\langle v, w\rangle+\langle v, z\rangle\right) \\
& \cdot \exp \{i\langle v, x\rangle\} d f(v) d m(w)
\end{aligned}
$$

for s-a.e. $x$ and $z$ in $C_{0}[0, T]$. Since $\int_{C_{0}[0, T]}\langle v, w\rangle d m(w)=0$, the Fubini theorem enables us to conclude that

$$
T_{\lambda}[G(x, \cdot)](z)=\int_{L_{2}[0, T]}\langle v, z\rangle \exp \{i\langle v, x\rangle\} d f(v)
$$

Since the right hand side of the last expression is independent of $\lambda$, we have

$$
T_{q}^{(p)}[G(x, \cdot)](z)=\int_{L_{2}[0, T]}\langle v, z\rangle \exp \{i\langle v, x\rangle\} d f(v)
$$

for s-a.e. $x$ and $z$ in $C_{0}[0, T]$.

Now considering Theorem 12 and applying repeatedly the first part of this proof, we obtain

$$
\begin{aligned}
& T_{q}^{(p)} {\left[\cdots T_{q}^{(p)}\left[\delta^{n} F(x, \cdot \ldots ; \cdot)\right]\left(z_{1}\right) \cdots\right]\left(z_{n}\right) } \\
& \quad=i^{n} \int_{L_{2}[0, T]}\left\langle v, z_{1}\right\rangle \cdots\left\langle v, z_{n}\right\rangle \exp \{i\langle v, x\rangle\} d f(v)
\end{aligned}
$$

for s-a.e. $x$ and $z_{1}, \ldots, z_{n}$ in $C_{0}[0, T]$. Finally by Theorem 12 again the proof is complete.

\section{Shifting Properties for Convolution Product}

We developed in Section 2 some properties relevant to shifting and computational rules for the Fourier-Feynman transform of functionals in the Banach algebra $\mathcal{S}$. In this section we study similar properties for the convolution product of functionals in $\mathcal{S}$. Let us begin with the definition of the convolution product of functionals on Wiener space.

Definition 17. Let $F$ and $G$ be functionals on $C_{0}[0, T]$. For $\lambda \epsilon$ $\mathbb{C}_{+}$and $y \in C_{0}[0, T]$, one defines the convolution product (if it exists) by

$$
\begin{aligned}
(F & * G)_{\lambda}(y) \\
& =\int_{C_{0}[0, T]}^{\mathrm{anw}_{\lambda}} F\left(\frac{y+x}{\sqrt{2}}\right) G\left(\frac{y-x}{\sqrt{2}}\right) d m(x), \\
(F & * G)_{q}(y) \\
& =\int_{C_{0}[0, T]}^{\operatorname{anf}_{q}} F\left(\frac{y+x}{\sqrt{2}}\right) G\left(\frac{y-x}{\sqrt{2}}\right) d m(x) .
\end{aligned}
$$

Obviously the convolution is bilinear in the sense that

$$
\begin{aligned}
{\left[\left(F_{1}+F_{2}\right) *\left(G_{1}+G_{2}\right)\right]_{q}(y) } \\
=\left(F_{1} * G_{1}\right)_{q}(y)+\left(F_{1} * G_{2}\right)_{q}(y) \\
\quad+\left(F_{2} * G_{1}\right)_{q}(y)+\left(F_{2} * G_{2}\right)_{q}(y)
\end{aligned}
$$

for all functionals $F_{i}, G_{i}$ on $C_{0}[0, T]$ for $i=1,2$, whenever each convolution exists.

Huffman et al. established the existence of the convolution product on $C_{0}[0, T]$ for functionals in $\mathcal{S}$.

Theorem 18 (Theorem 3.2 of [5]). Let $F$ and $G$ be elements of $\mathcal{S}$ with corresponding finite Borel measures $f$ and $g$, respectively. Then their convolution product $(F * G)_{q}$ exists and is given by the formula

$$
\begin{gathered}
(F * G)_{q}(y)=\int_{L_{2}^{2}[0, T]} \exp \left\{\frac{i}{\sqrt{2}}\langle v+w, y\rangle\right. \\
\left.-\frac{i}{4 q}\|v-w\|^{2}\right\} d f(v) d g(w)
\end{gathered}
$$

for s-a.e. $y \in C_{0}[0, T]$.

Our first result in this section is a relationship between time shifting and frequency shifting of the convolution product on Wiener space.

Theorem 19. Let $F$ and $G$ be functionals on $C_{0}[0, T]$ and let $x_{0} \in C_{0}[0, T]$. Then one has

$$
\left[F\left(\cdot-x_{0}\right) * G\left(\cdot-x_{0}\right)\right]_{q}(y)=(F * G)_{q}\left(y-\sqrt{2} x_{0}\right)
$$

if each side exists.

Proof. For all $\lambda>0$ and for s-a.e. $y \in C_{0}[0, T]$, we have

$$
\begin{aligned}
& {\left[F\left(\cdot-x_{0}\right) * G\left(\cdot-x_{0}\right)\right]_{\lambda}(y)} \\
& \quad=\int_{C_{0}[0, T]} F\left(\frac{y+\lambda^{-1 / 2} x}{\sqrt{2}}-x_{0}\right) \\
& \quad \cdot G\left(\frac{y-\lambda^{-1 / 2} x}{\sqrt{2}}-x_{0}\right) d m(x)=(F * G)_{\lambda} \\
& \quad \cdot\left(y-\sqrt{2} x_{0}\right)
\end{aligned}
$$

if the Wiener integrals exist. Hence we have the result. 
The following theorem is reminiscent of the time shifting theorem for Fourier-Feynman transform (Theorem 4) in Section 2. But in this theorem we have to shift back $x_{0}$ for $F$ and shift front $x_{0}$ for $G$ to obtain a concrete form of a time shifting formula for the convolution product.

Theorem 20 (time shifting). Let $F$ and $G$ be given as in Theorem 18 and let $x_{0} \in C_{0}[0, T]$. Then one has

$$
\begin{aligned}
& {\left[F\left(\cdot-x_{0}\right) * G\left(\cdot+x_{0}\right)\right]_{q}(y)=\exp \left\{i q\left\|x_{0}\right\|^{2}\right\}} \\
& \cdot\left[F(\cdot) \exp \left\{i q\left\langle x_{0}, \cdot\right\rangle\right\} * G(\cdot) \exp \left\{-i q\left\langle x_{0}, \cdot\right\rangle\right\}\right]_{q} \\
& \cdot(y)
\end{aligned}
$$

for s-a.e. $y \in C_{0}[0, T]$.

Proof. Let $F_{0}(x)=F\left(x-x_{0}\right)$ and $G_{0}(x)=G\left(x+x_{0}\right)$. By (5) we have

$$
\begin{aligned}
F_{0}(x) & =\int_{L_{2}[0, T]} \exp \{i\langle v, x\rangle\} d f_{0}(v), \\
G_{0}(x) & =\int_{L_{2}[0, T]} \exp \{i\langle w, x\rangle\} d g_{0}(w),
\end{aligned}
$$

where $f_{0}(E)=\int_{E} \exp \left\{-i\left\langle v, x_{0}\right\rangle\right\} d f(v)$ and $g_{0}(E)=\int_{E} \exp \{i\langle w$, $\left.\left.x_{0}\right\rangle\right\} d g(w)$ for a Borel subset $E$ of $L_{2}[0, T]$. Then, by Theorem 18 , the left hand side of (60) is given by

$$
\begin{aligned}
& \left(F_{0} * G_{0}\right)_{q}(y)=\int_{L_{2}^{2}[0, T]} \exp \left\{\frac{i}{\sqrt{2}}\langle v+w, y\rangle\right. \\
& \left.-\frac{i}{4 q}\|v-w\|^{2}\right\} d f_{0}(v) d g_{0}(w) \\
& =\int_{L_{2}^{2}[0, T]} \exp \left\{\frac{i}{\sqrt{2}}\langle v+w, y\rangle-i\left\langle v-w, x_{0}\right\rangle\right. \\
& \left.-\frac{i}{4 q}\|v-w\|^{2}\right\} d f(v) d g(w)
\end{aligned}
$$

for s-a.e. $y \in C_{0}[0, T]$.

To consider the right hand side of (60), let

$$
\begin{aligned}
F_{1}(x) & =F(x) \exp \left\{i q\left\langle x_{0}, x\right\rangle\right\} \\
& =\int_{L_{2}[0, T]} \exp \left\{i\left\langle v_{1}, x\right\rangle\right\} d f_{1}\left(v_{1}\right), \\
G_{1}(x) & =G(x) \exp \left\{-i q\left\langle x_{0}, x\right\rangle\right\} \\
& =\int_{L_{2}[0, T]} \exp \left\{i\left\langle w_{1}, x\right\rangle\right\} d g_{1}\left(w_{1}\right),
\end{aligned}
$$

where $f_{1}(E)=f\left(E-q x_{0}\right)$ and $g_{1}(E)=g\left(E+q x_{0}\right)$ for a Borel subset $E$ of $L_{2}[0, T]$. Then, by Theorem 18, we have

$$
\begin{aligned}
& \left(F_{1} * G_{1}\right)_{q}(y)=\int_{L_{2}^{2}[0, T]} \exp \left\{\frac{i}{\sqrt{2}}\left\langle v_{1}+w_{1}, y\right\rangle\right. \\
& \left.-\frac{i}{4 q}\left\|v_{1}-w_{1}\right\|^{2}\right\} d f_{1}\left(v_{1}\right) d g_{1}\left(w_{1}\right) \\
& =\int_{L_{2}^{2}[0, T]} \exp \left\{\frac{i}{\sqrt{2}}\langle v+w, y\rangle\right. \\
& \left.-\frac{i}{4 q}\left\|v-w+2 q x_{0}\right\|^{2}\right\} d f(v) d g(w)
\end{aligned}
$$

for s-a.e. $y \in C_{0}[0, T]$. Since $\left\|v-w+2 q x_{0}\right\|^{2}=\|v-w\|^{2}+$ $4 q\left\langle v-w, x_{0}\right\rangle+4 q^{2}\left\|x_{0}\right\|^{2}$, we have

$$
\begin{aligned}
& \left(F_{1} * G_{1}\right)_{q}(y)=\exp \left\{-i q\left\|x_{0}\right\|^{2}\right\} \\
& \cdot \int_{L_{2}^{2}[0, T]} \exp \left\{\frac{i}{\sqrt{2}}\langle v+w, y\rangle-i\left\langle v-w, x_{0}\right\rangle\right. \\
& \left.-\frac{i}{4 q}\|v-w\|^{2}\right\} d f(v) d g(w)
\end{aligned}
$$

for s-a.e. $y \in C_{0}[0, T]$ and this completes the proof.

Considering the second part of the proof of Theorem 20 above, we see that, for $F$ and $G$ given as in Theorem 18,

$$
\begin{aligned}
& {\left[F(\cdot) \exp \left\{i q\left\langle x_{0}, \cdot\right\rangle\right\} * G(\cdot) \exp \left\{i q\left\langle x_{0}, \cdot\right\rangle\right\}\right]_{q}(y)} \\
& \quad=\int_{L_{2}^{2}[0, T]} \exp \left\{\frac{i}{\sqrt{2}}\left\langle v+w+2 q x_{0}, y\right\rangle\right. \\
& \left.-\frac{i}{4 q}\|v-w\|^{2}\right\} d f(v) d g(w) \\
& \quad=\exp \left\{i \sqrt{2} q\left\langle x_{0}, y\right\rangle\right\}(F * G)_{q}(y)
\end{aligned}
$$

for s-a.e. $y \in C_{0}[0, T]$.

The following is a scaling theorem for the convolution product.

Theorem 21 (scaling). Let $F$ and $G$ be given as in Theorem 18 . If $a$ is a nonzero real number, then

$$
[F(a \cdot) * G(a \cdot)]_{q}(y)=(F * G)_{q / a^{2}}(a y)
$$

for s-a.e. $y \in C_{0}[0, T]$.

Proof. By the same method as used in the proof of Theorem 7 we have

$$
\begin{aligned}
& {[F(a \cdot) * G(a \cdot)]_{q}(y)=\int_{L_{2}^{2}[0, T]} \exp \left\{\frac{i}{\sqrt{2}}\langle v+w, a y\rangle\right.} \\
& \left.-\frac{a^{2} i}{4 q}\|v-w\|^{2}\right\} d f(v) d g(w)
\end{aligned}
$$

for s-a.e. $y \in C_{0}[0, T]$. Hence by Theorem 18 we obtain the result. 
Our next corollary follows immediately from the scaling theorem above by putting $a=-1$. This result is called time reversal because we replace $x$ by $-x$ in $F(x)$ and $G(x)$ to get $F(-x)$ and $G(-x)$, respectively. The convolution of these new functionals is obtained by simply replacing $y$ by $-y$ in the convolution of $F(x)$ and $G(x)$.

Corollary 22 (time reversal). Let $F$ and $G$ be given as in Theorem 18. Then one has

$$
[F(-\cdot) * G(-\cdot)]_{q}(y)=(F * G)_{q}(-y)
$$

for s-a.e. $y \in C_{0}[0, T]$.

Our next theorem is useful to obtain the convolution product of new functionals from the convolution product of old functionals when we know their convolution product.

Theorem 23 (modulation). Let $F$ and $G$ be given as in Theorem 18 and let $x_{0} \in C_{0}[0, T]$. Then

$$
\begin{aligned}
& {\left[F(\cdot) \cos \left(q\left\langle x_{0}, \cdot\right\rangle\right) * G(\cdot) \cos \left(q\left\langle x_{0}, \cdot\right\rangle\right)\right]_{q}(y)} \\
& =\frac{1}{4}\left(K[F, G]\left(1,0,0 ; x_{0}, y\right)\right. \\
& +K[F, G]\left(-1,0,0 ; x_{0}, y\right) \\
& +K[F, G]\left(0,1,1 ; x_{0}, y\right) \\
& \left.+K[F, G]\left(0,1,-1 ; x_{0}, y\right)\right) \text {, } \\
& {\left[F(\cdot) \cos \left(q\left\langle x_{0}, \cdot\right\rangle\right) * G(\cdot) \sin \left(q\left\langle x_{0}, \cdot\right\rangle\right)\right]_{q}(y)} \\
& =\frac{1}{4 i}\left(K[F, G]\left(1,0,0 ; x_{0}, y\right)\right. \\
& \text { - } K[F, G]\left(-1,0,0 ; x_{0}, y\right) \\
& \text { - } K[F, G]\left(0,1,1 ; x_{0}, y\right) \\
& \left.+K[F, G]\left(0,1,-1 ; x_{0}, y\right)\right) \text {, } \\
& {\left[F(\cdot) \sin \left(q\left\langle x_{0}, \cdot\right\rangle\right) * G(\cdot) \cos \left(q\left\langle x_{0}, \cdot\right\rangle\right)\right]_{q}(y)} \\
& =\frac{1}{4 i}\left(K[F, G]\left(1,0,0 ; x_{0}, y\right)\right. \\
& \text { - } K[F, G]\left(-1,0,0 ; x_{0}, y\right) \\
& +K[F, G]\left(0,1,1 ; x_{0}, y\right) \\
& \text { - } \left.K[F, G]\left(0,1,-1 ; x_{0}, y\right)\right) \text {, } \\
& {\left[F(\cdot) \sin \left(q\left\langle x_{0}, \cdot\right\rangle\right) * G(\cdot) \sin \left(q\left\langle x_{0}, \cdot\right\rangle\right)\right]_{q}(y)} \\
& =-\frac{1}{4}\left(K[F, G]\left(1,0,0 ; x_{0}, y\right)\right. \\
& +K[F, G]\left(-1,0,0 ; x_{0}, y\right) \\
& \text { - } K[F, G]\left(0,1,1 ; x_{0}, y\right) \\
& \text { - } \left.K[F, G]\left(0,1,-1 ; x_{0}, y\right)\right) \text {, }
\end{aligned}
$$

for s-a.e. $y \in C_{0}[0, T]$.

Proof. Since $\left[F\left(\cdot-\gamma x_{0}\right) * G\left(\cdot+\gamma x_{0}\right)\right]_{q}(y) \equiv 1$ for $F \equiv G \equiv 1$, by the modulation property, Theorem 23, and Euler's formula, the results follow immediately.

Finally by (60) and (68) we obtain (72). Using $\sin \left(q\left\langle x_{0}, \cdot\right\rangle\right)=$ $(1 / 2 i)\left(\exp \left\{i q\left\langle x_{0}, \cdot\right\rangle\right\}-\exp \left\{-i q\left\langle x_{0}, \cdot\right\rangle\right\}\right)$ the other conclusions are proved similarly.

Since the Dirac measure concentrated at $v=0$ in $L_{2}[0, T]$ is a complex Borel measure, the constant function $F \equiv 1$ belongs to $\mathcal{S}$. Hence we have the following corollary.

Corollary 24. Let $x_{0} \in C_{0}[0, T]$. Then one has

$$
\begin{aligned}
& {\left[\cos \left(q\left\langle x_{0}, \cdot\right\rangle\right) * \cos \left(q\left\langle x_{0}, \cdot\right\rangle\right)\right]_{q}(y)} \\
& =\frac{1}{2}\left(\cos \left(\sqrt{2} q\left\langle x_{0}, y\right\rangle\right)+\exp \left\{-i q\left\|x_{0}\right\|^{2}\right\}\right), \\
& {\left[\cos \left(q\left\langle x_{0}, \cdot\right\rangle\right) * \sin \left(q\left\langle x_{0}, \cdot\right\rangle\right)\right]_{q}(y)} \\
& =\left[\sin \left(q\left\langle x_{0}, \cdot\right\rangle\right) * \cos \left(q\left\langle x_{0}, \cdot\right\rangle\right)\right]_{q}(y) \\
& =\frac{1}{2} \sin \left(\sqrt{2} q\left\langle x_{0}, y\right\rangle\right), \\
& {\left[\sin \left(q\left\langle x_{0}, \cdot\right\rangle\right) * \sin \left(q\left\langle x_{0}, \cdot\right\rangle\right)\right]_{q}(y)} \\
& \quad=-\frac{1}{2}\left(\cos \left(\sqrt{2} q\left\langle x_{0}, y\right\rangle\right)-\exp \left\{-i q\left\|x_{0}\right\|^{2}\right\}\right)
\end{aligned}
$$

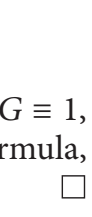




\section{Conflict of Interests}

The author declares that there is no conflict of interests regarding the publication of this paper.

\section{Acknowledgment}

This research was supported by Basic Science Research Program through the National Research Foundation of Korea (NRF) funded by the Ministry of Education, Science and Technology (2010-0022563).

\section{References}

[1] M. D. Brue, A functional transform for Feynman integrals similar to the Fourier transform [Ph.D. thesis], University of Minnesota, Minneapolis, Minn, USA, 1972.

[2] R. H. Cameron and D. A. Storvick, "An $L_{2}$ analytic FourierFeynman transform," The Michigan Mathematical Journal, vol. 23, no. 1, pp. 1-30, 1976.

[3] G. W. Johnson and D. L. Skoug, "An $L_{p}$ analytic FourierFeynman transform," The Michigan Mathematical Journal, vol. 26, no. 1, pp. 103-127, 1979.

[4] T. Huffman, C. Park, and D. Skoug, "Analytic Fourier-Feynman transforms and convolution," Transactions of the American Mathematical Society, vol. 347, no. 2, pp. 661-673, 1995.

[5] T. Huffman, C. Park, and D. Skoug, "Convolutions and FourierFeynman transforms of functionals involving multiple integrals," Michigan Mathematical Journal, vol. 43, no. 2, pp. 247261, 1996.

[6] B. J. Kim, B. S. Kim, and I. Yoo, "Change of scale formulas for wiener integrals related to Fourier-Feynman transform and convolution," Journal of Function Spaces, vol. 2014, Article ID 657934, 7 pages, 2014.

[7] D. Skoug and D. Storvick, "A survey of results involving transforms and convolutions in function space," Rocky Mountain Journal of Mathematics, vol. 34, no. 3, pp. 1147-1175, 2004.

[8] G. Johnson and D. Skoug, "Scale-invariant measurability in Wiener space," Pacific Journal of Mathematics, vol. 83, no. 1, pp. 157-176, 1979.

[9] R. H. Cameron and D. A. Storvick, "Some Banach algebras of analytic Feynman integrable functionals," in Analytic Functions Kozubnik 1979, vol. 798 of Lecture Notes in Mathematics, pp. 1867, Springer, Berlin, Germany, 1980.

[10] R. P. Feynman and A. R. Hibbs, Quantum Mechanics and Path Integrals, McGraw-Hill, New York, NY, USA, 1965.

[11] R. P. Feynman, "Space-time approach to non-relativistic quantum mechanics," Reviews of Modern Physics, vol. 20, no. 2, pp. 367-387, 1948.

[12] K. S. Chang, G. W. Johnson, and D. L. Skoug, "The Feynman integral of quadratic potentials depending on two time variables," Pacific Journal of Mathematics, vol. 122, no. 1, pp. 11-33, 1986.

[13] P. V. O'Neil, Advanced Engineering Mathematics, Thomson, 5th edition, 2003.

[14] R. H. Cameron and D. A. Storvick, "A new translation theorem for the analytic Feynman integral," Revue Roumaine de Mathématique Pures et Appliquées, vol. 27, no. 9, pp. 937-944, 1982.
[15] J. M. Ahn, K. S. Chang, B. S. Kim, and I. Yoo, "Fourier-Feynman transform, convolution and first variation," Acta Mathematica Hungarica, vol. 100, no. 3, pp. 215-235, 2003.

[16] C. Park, D. Skoug, and D. Storvick, "Relationships among the first variation, the convolution product, and the FourierFeynman transform," Rocky Mountain Journal of Mathematics, vol. 28, no. 4, pp. 1447-1468, 1998. 


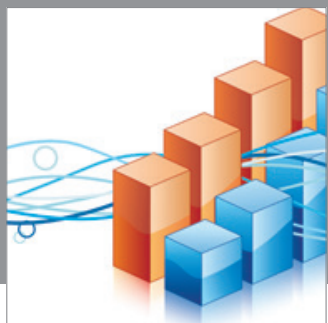

Advances in

Operations Research

mansans

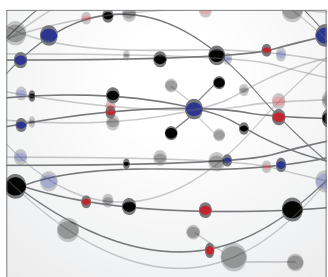

The Scientific World Journal
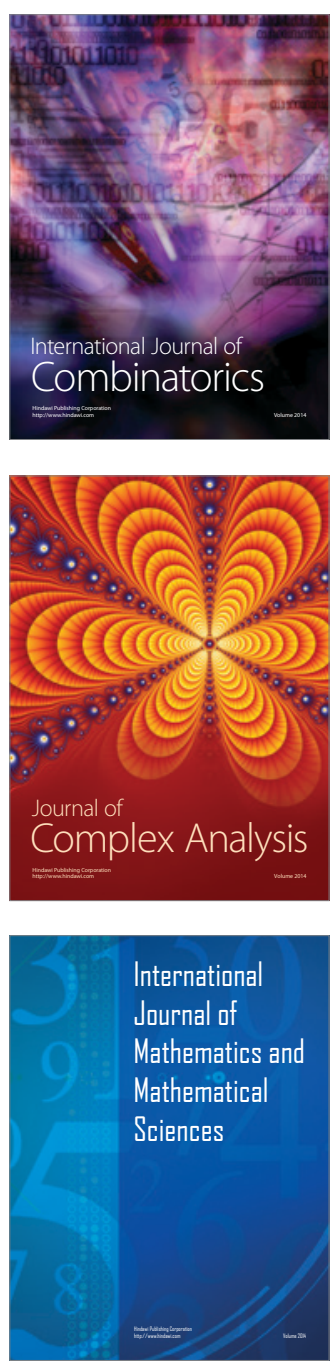
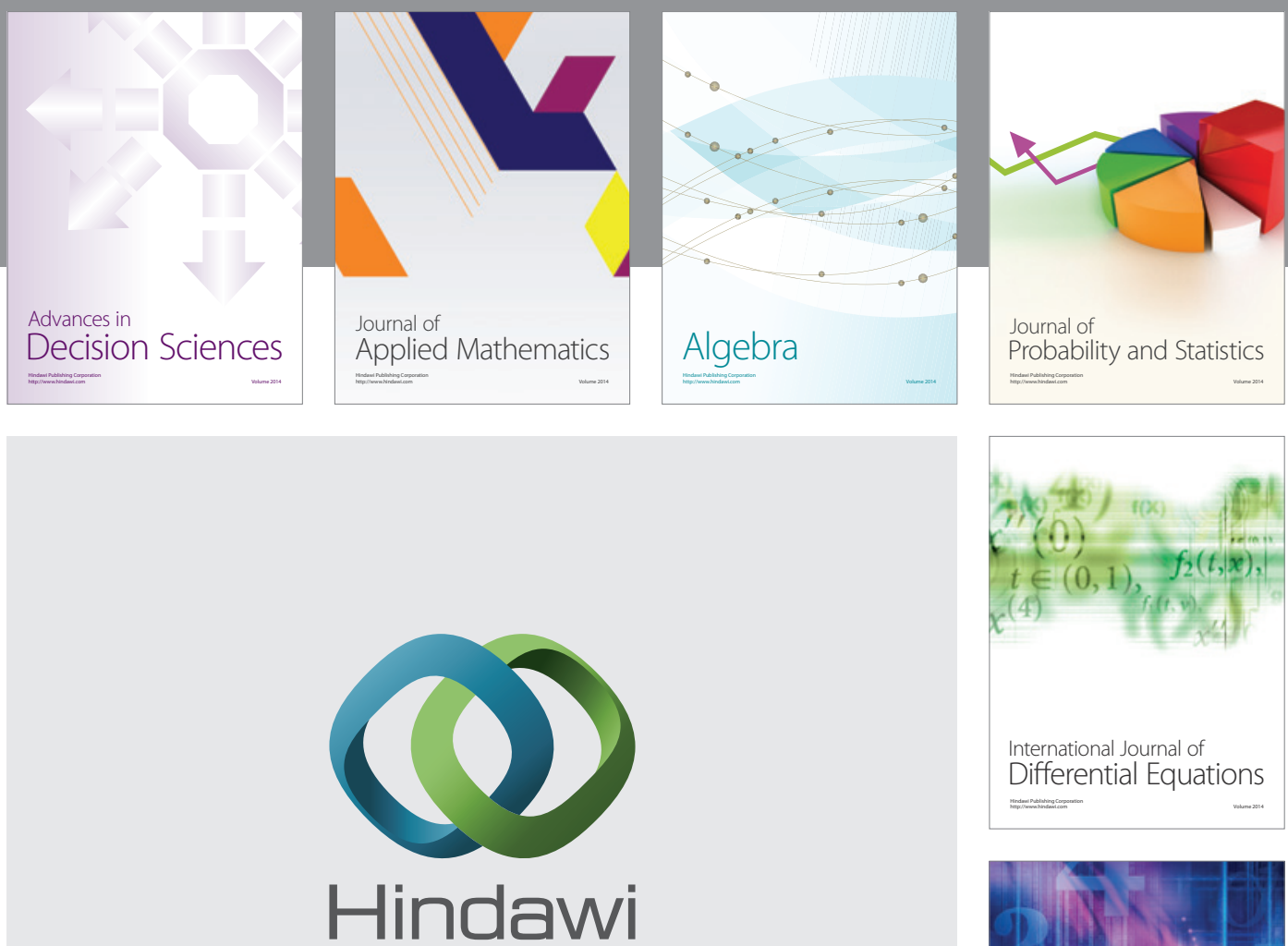

Submit your manuscripts at http://www.hindawi.com
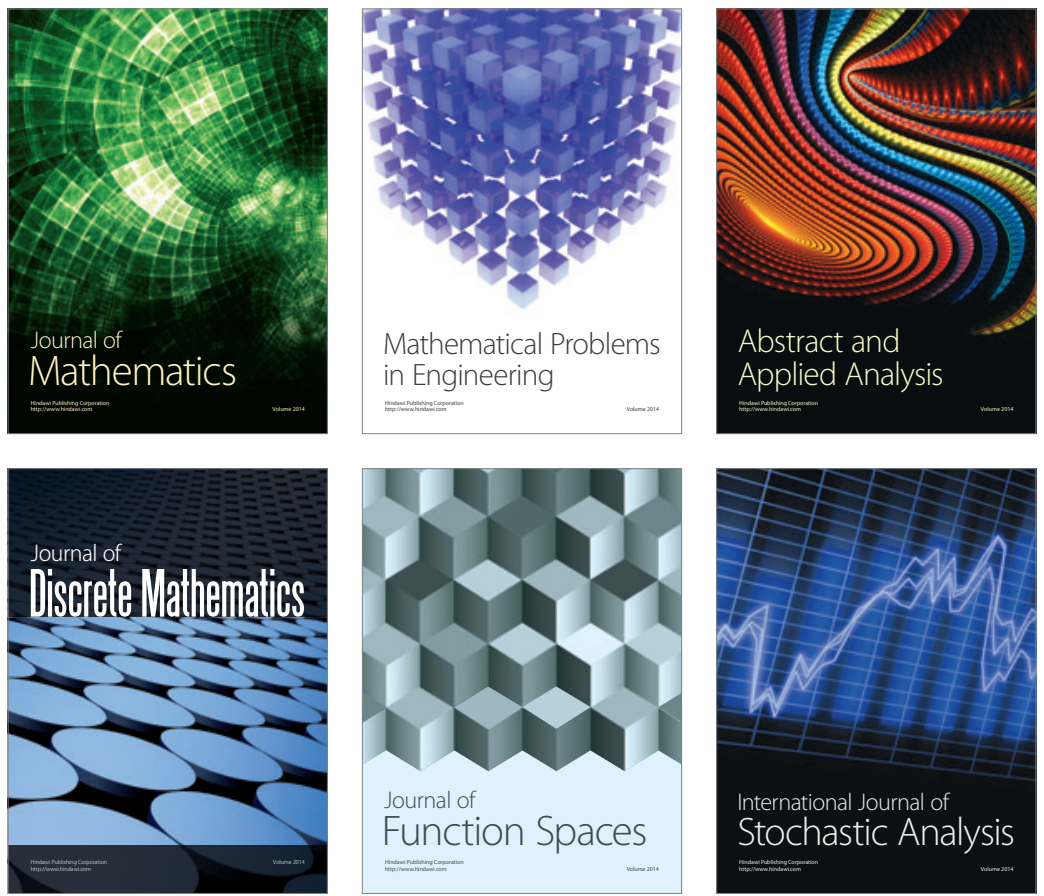

Journal of

Function Spaces

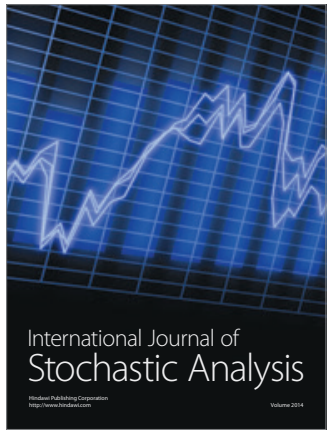

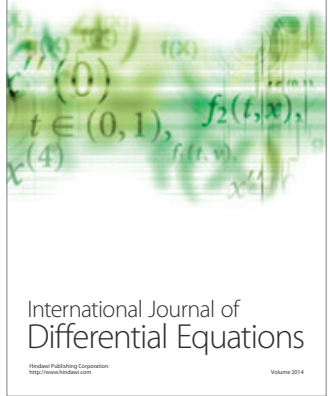
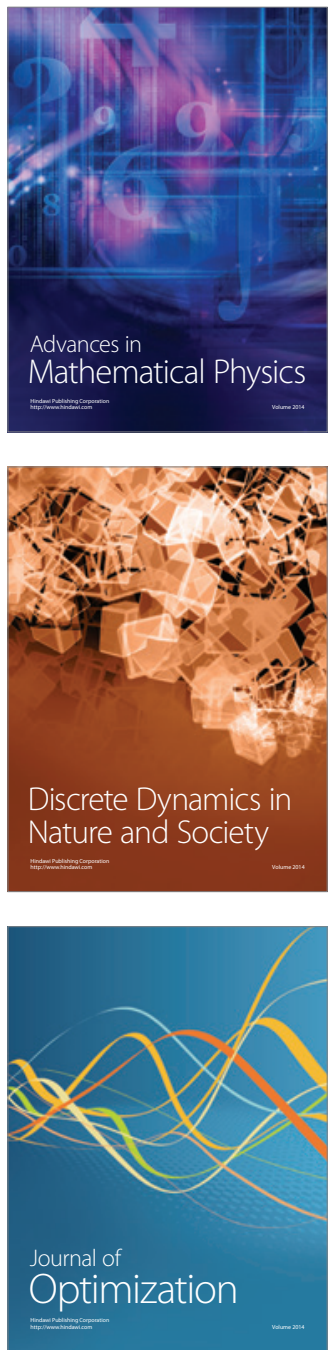